\title{
Design and Implementation of High Directivity Directional Coupler at VHF Band Using Grounding Composed of Strip Lines Technics
}

\author{
Atef Merghani Abdallah Abdalmagd *§ \\ *Atyaf Industrial Complex, Sudan \\ §a_marghani@hotmail.com,
}

\begin{abstract}
This paper proposes a design of high directivity directional coupler (D.C) based on grounding composed of strip lines for high power Radar transmitter at Very High Frequency (VHF), (150 $200 \mathrm{MHz}$ ). The directional coupler is used to check and verify the transmitter output high power, frequency, and reflected signal from an antenna connected with the Radar transmitter. The performance requirements of directional couplers are a strong coupling to reduce the effect on the transmitted output power and high directivity to suppress the interference of the reflected signal from the antenna. So far, various architectures have been proposed to gain high directivity, and there have been many studies used to obtain a strong coupling and higher directivity. However, the conventional architecture of the directional coupler has a directivity of only about $20 \mathrm{~dB}$, and there have been difficulties to achieve the higher directivity of more than $20 \mathrm{~dB}$. In this paper, the proposed architecture of directional coupler based on grounding composed of strip lines is discussed and compares the test results of the proposed directional coupler with the conventional one. The high directivity directional coupler is designed using a computer-aided design Simulation program; Advance Design System (ADS 2016), using Rogers 4003 substrate. The directional coupler was fabricated on printed circuit board (PCB) technology and measured using a vector network analyzer (VNA). The results show that the proposed directional coupler has directivity between $-25 t o-24 d B$ inside the working bandwidth and is adequate for a high-power radar transmitter.
\end{abstract}

Key words-Directional Coupler, High Directivity, High Power Amplifier, Solid-State Power Amplifier, Vector Network Analyzer.

\section{INTRODUCTION}

Directional couplers (D.C) are passive devices most frequently used to sample the output of a power amplifier stage, to control and monitor its forward power level. Other use of directional couplers are for detecting antenna faults, measuring unknown impedances, and combining signals. Important directional coupler properties are low insertion loss and high directivity, which is a measure of the couplers capability to detect power flow in only one direction [1].

There are many different ways of forming directional couplers [1]-[4]. They can be constructed in stripline, Microstrip, coaxial, and even waveguide. One of the most common forms of conventional directional coupler is shown in Fig.1. It consists of two transmission lines placed close together along the axes of propagation. If a signal is an incident at input port P1; P2 is the through port, and the coupled wave exits at P3. With the proper design and proper terminations no signal exits at $\mathrm{P} 4$ (the isolated port). The directional couplers in microwave systems can be classified into hybrid and Strip-line structures. The hybrid type has a good coupling property, but its frequency range is narrow for the directivity property. On the other hand, the directivity of the strip-line structure has a wider bandwidth in frequency. However, it is difficult to get more than $20 \mathrm{~dB}$ for directivity property.

Therefore, this paper is focused on a study showing that the proposed architecture based on the strip-line structure can achieve $25-24 \mathrm{~dB}$ for directivity. The proposed stripline structure composed of the same materials can make equal mode phase velocities in the coupler. To evaluate the

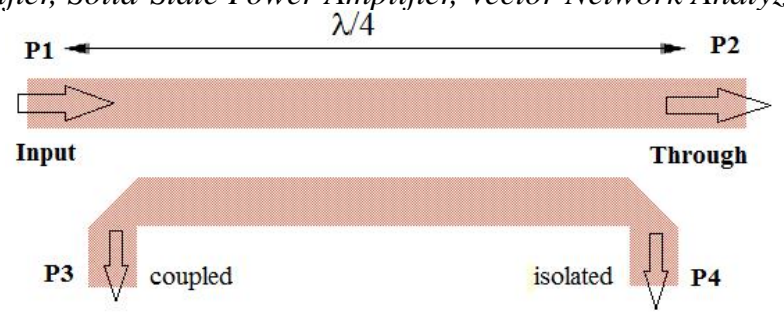

Fig. 1. Conventional directional coupler

performance of the proposed strip-line structure, its directivity has been monitored with simulations and experiments, and the factors affecting the directivity are analyzed in this paper. Furthermore, this paper suggests a new design method by adding a ground between the discontinuous bands and step in the coupler by comparing the simulation results to the properties of output frequency from the directional coupler with parallel coupled strip-line. A comparison between the experimental results for the coupler applied with the proposed design and conventional couplers are also shown in this paper.

\section{Design Theory of Directional Coupler Using PARALlel COUPLED STRIP-LINE}

When two transmission lines (TL) are brought near each other, as shown in Fig.2, power can be coupled from one TL to another one. For coupled line directional couplers, this coupling between TLs is a useful phenomenon and is the 


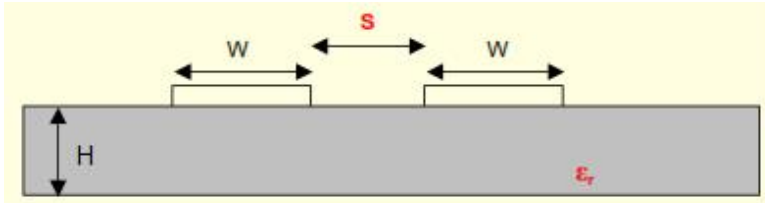

Fig. 2. Uniform coupler with coupled line

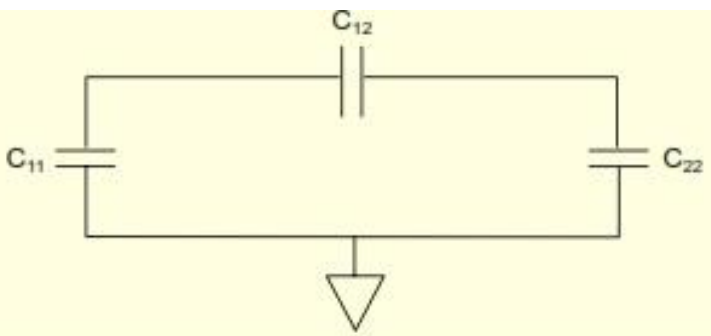

Fig. 3. Equivalent circuit of uniform coupler with coupled line

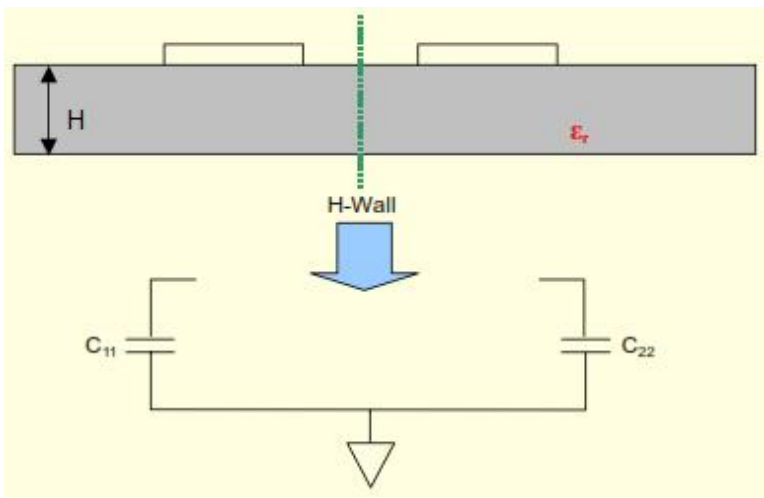

Fig. 4. Equivalent circuit for even mode

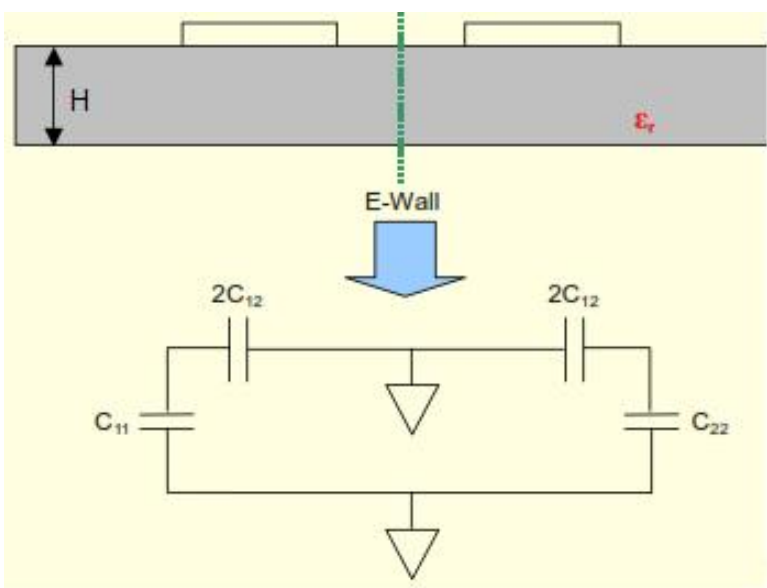

Fig. 5. Equivalent circuit for odd mode

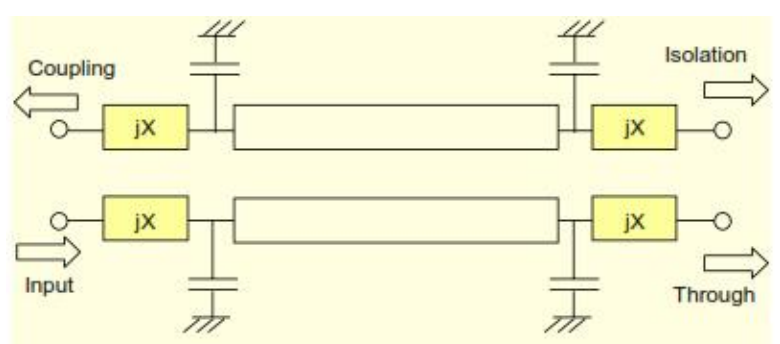

Fig. 6. Equivalent circuit of a directional coupler

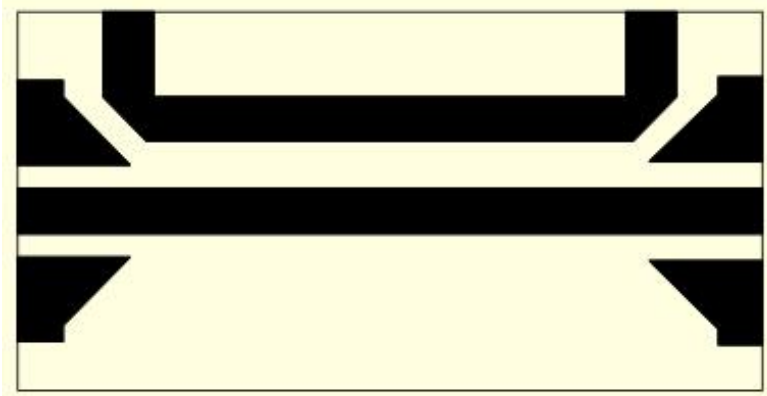

Fig. 7. The Proposed architecture of high directivity directional coupler

physical principle upon which the couplers are based.

Consider the geometry shown in Fig.2, when voltages are applied to the edges of TLs, charge distribution will be induced on all of the conductors. The voltages and total charge are related to each other through capacitance $C i j$ as illustrated in Fig.3.

To simplify the analysis, assume the two strip lines are identical and located on top of the dielectric. Because of the symmetry, we can use an even-odd mode solution approach as shown in Fig.4 and Fig.5, respectively.

In Eq.1, the characteristic impedance $Z_{0 e}$ using the capacitances for the even mode, When equivalent even mode capacitance $C_{e}=C 11=C 22$ then [1]:

$$
Z_{0 e}=\sqrt{\frac{L}{C_{e}}}=\frac{\sqrt{L C_{e}}}{C_{e}}=\frac{1}{v C_{e}}
$$

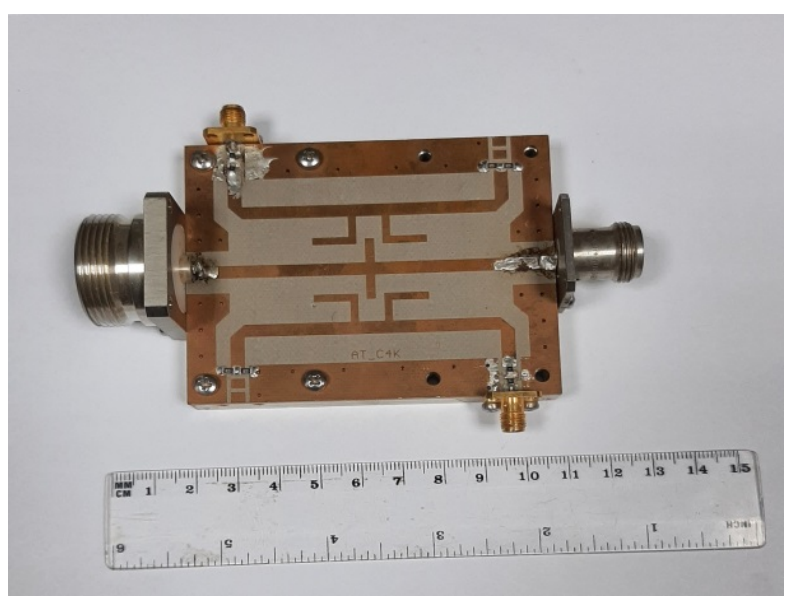

Fig. 8. VHF high directivity directional couplerr 
TABLE I

The Physical Dimensions of Directional Coupler

\begin{tabular}{llll}
\hline $\mathrm{C}(\mathrm{dB})$ & $\mathrm{W}(\mathrm{mm})$ & $\mathrm{S}(\mathrm{mm})$ & $\mathrm{L}(\mathrm{mm})$ \\
\hline$>30$ & 3.34681 & 12.4875 & 88 \\
\hline
\end{tabular}

Where $v$ is the radio wave speed $(\mathrm{m} / \mathrm{sec}$ ). Eq. 2 shows the oddmode characteristic $Z 0_{o}$ impedance using the capacitances for the odd mode $C_{o}$ :

$$
C_{0}=C_{11}+2 C_{12}=C_{22}+2 C_{21} \rightarrow Z o_{0}=\frac{1}{v C_{e}}
$$

In a case where the distance between two transmission lines is closed as the occurrence of the coupling, each of the even and odd modes has characteristic impedance in equations Eq.1 and Eq.2 different from the other one. On the other hand, the characteristic impedances of the even and odd modes come to be the same in relatively far enough distances. The coupling coefficient $(C)$, isolating coefficient $(I)$, and transmission coefficient $(T)$ can be described in Eq.3, with output against input to the coupling or isolating port of a directional coupler with parallel coupled strip line shown in Fig.1.

$$
\begin{gathered}
C(d B)=-10 \log \left|\frac{P_{3}}{P_{1}}\right|, \\
I(d B)=-10 \log _{10}\left|\frac{P_{4}}{P_{1}}\right|, \\
T(d B)=-10 \log _{10}\left|\frac{P_{2}}{P_{1}}\right|,
\end{gathered}
$$

The directivity $(D)$, which is the most important of the performance indices, is defined in Eq.4 as the relative power of the isolating port for the power of the coupling port. Then, the directivity in $\mathrm{dB}$ is described as follows:

$$
D(d B)=I(d B)-C(d B)
$$

In this paper, parallel coupled strip-line architecture with transverse electromagnetic wave transmission mode has been applied to improve the directivity. In general, it is hard to get high directivity in a directional coupler having a weakened coupling coefficient. Therefore, a directional coupler having a stronger coupling coefficient has been designed on Rogers 4003 substrate with relative permittivity $\left(\epsilon_{r}\right)$ is 3.55 , while dissipation factor $(\tan \delta)$ is 0.0027 , also the thickness for this substrate $(H)$ is $1.52 \mathrm{~mm}$, and thickness of conductor copper layer $(T)$ is $35 \mathrm{~m}$. As for the range of the measurement frequency, the central frequency has been set $175 \mathrm{MHz}$ experimented with a range of $50 \mathrm{MHz}$ bandwidth. The physical dimension of TLs width $(W)$, length $(L)$, and the space between $T L s(S)$ for the coupler was obtained by a simulator for the specified frequency and coupling are illustrated in Table II.

\section{Design of High DiRectivity DiRectional COUPLER}

The high directivity generally indicates directivity of more than $20 \mathrm{~dB}$. The conventional directional couplers using parallel coupled strip-line have a directivity of about $20 \mathrm{~dB}$.
TABLE II

COMPARISON BETWEEN CONVENTIONAL \& PROPOSED DIRECTIONAL COUPLERS (D.C) RESULTS

\begin{tabular}{lllll}
\hline & \multicolumn{2}{l}{ Conventional D.C } & \multicolumn{2}{l}{ The Proposed D.C } \\
\hline Frequency MHz & 150 & 200 & 150 & 200 \\
Coupling dB & -36.991 & -34.483 & -46.795 & -44.313 \\
Insertion loss dB & -50.573 & -47.683 & -25.855 & -23.027 \\
Return loss dB & -0.018 & -0.022 & -0.034 & -0.030 \\
Isolation dB & -57.127 & -53.480 & -72.001 & -68.420 \\
Directivity dB & -20.182 & -18.997 & -25.206 & -24.107 \\
\hline
\end{tabular}

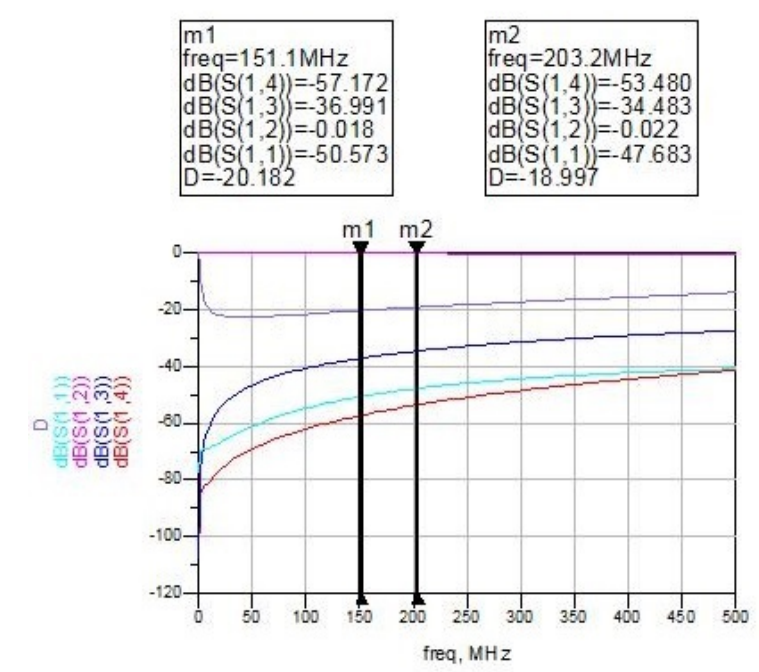

Fig. 9. Experimental results of conventional directional coupler

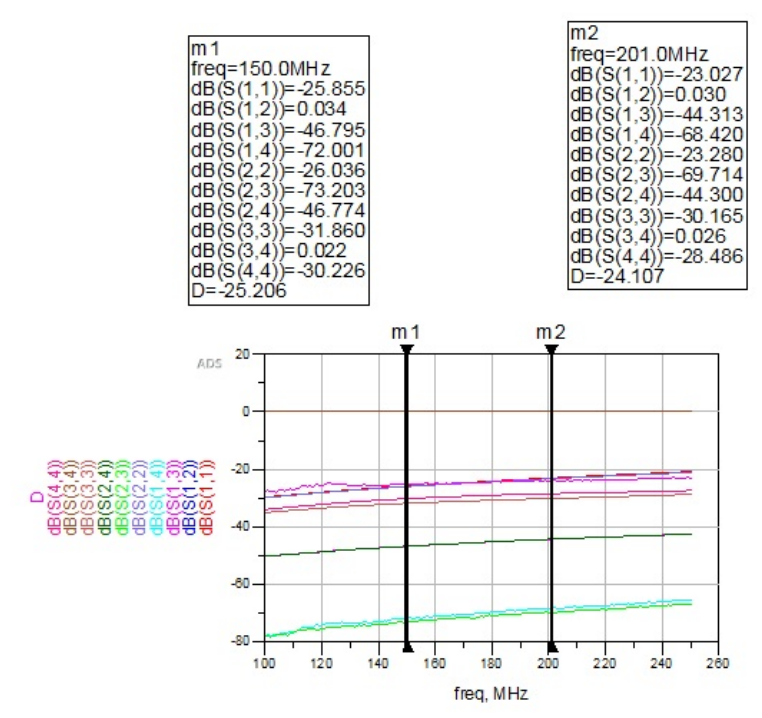

Fig. 10. Practical results of proposed high directivity directional coupler 
The 1st Scientific Conference for Aerospace Researches \& Applications Sudan (SCARA-1)-2021

Therefore, a new architecture or design method is required to improve the directivity. The capacitance, inductance, and impedance representing the properties of a transmission line are easily obtained for a single transmission line. However, practical circuits are composed of transmission lines with complex structures. In this case, the property of each transmission line is made up of complex relationships among line materials according to signal layer; isolation materials between signal layers; shape, including thickness, area, and length of the transmission line; and the position state of adjacent transmission lines. The characteristic of the directional coupler is determined by the characteristic parameters of such capacitance and inductance that are dependent on the structure of the transmission lines [1]-[3]. To design high directivity directional couplers the signal quantity at the coupling port must be reduced by preventing a coupling effect between 50 $\Omega$ transmission lines. The result in a design method for the compensation of the impedance mismatching due to the discontinuous reactant components by the capacitance generated between line and ground [6] as shown in Fig.6.

This paper proposes architecture to compensate impedance mismatching by adding a grounding area near the ports, with the capacitance between line and ground as shown in Fig.7. Also, segments of TLs were shaping in the center of the coupler for improving the coupling coefficient. The capacitance formed on the grounding area was calculated as follows:

$$
C=\frac{8.85 \times 10^{-12} \times \epsilon_{r} \times d}{S}
$$

Where $\epsilon_{r}$ relative permittivity of the substrate, $\mathrm{d}$ is the grounding area $\left(m^{2}\right), S$ is the distance $(m)$ between transmission line and ground, and the constant represent the free space permittivity.

Fig. 8 shows the $(50 \times 90) \mathrm{mm}$ fabricated architecture of high directivity directional coupler for (VHF) radar using ground composed technic. The shape in the center of the coupler was designed by the practical experiences, and used to improve the coupling coefficient; on the other hand, it decreases the reflection coefficient but still was good enough for high power usage.

\section{RESUlts And Discussions}

Fig.9 shows the experimental results of the conventional directional coupler. The isolation coefficient $S(1,4)$, coupling coefficient $S(1,3)$, transmission coefficient $\mathbf{S}(1,2)$, reflection coefficient $\mathbf{S}(1,1)$, and directivity $(D)$ are obtained by the experiments on the designed conventional directional coupler described in Fig.1. The directivity was between 20 to $19 \mathrm{~dB}$ inside the bandwidth.

The vector network analyzer was used to measure the S-parameters. The practical results of the proposed high directivity directional coupler with grounding area described in Fig.8. All files of measured S-parameters are collected in one file (touchstone file) to draw S-parameters in the same graph using ADS (2016) design software, as shown in Fig.10. Fig.10 shows the results of isolation and coupling coefficients are between -72 to $-68 \mathrm{~dB}$ and -47 to $-44 \mathrm{~dB}$, respectively. According to Eq.4, its clear the directional coupler in which the grounding area is deposited can get directivity between -25 to -24 dB. Fig. 10 also shows that the insertion loss at the passing port is not greater than $-0.034 \mathrm{~dB}$ and reflection losses at all ports are not greater than $-23 \mathrm{~dB}$. Table II summarizes the comparison between the simulation results of the conventional directional coupler and the proposed high directivity directional coupler using grounding composed technic. The proposed directional coupler can achieve a higher directivity compared with the conventional directional couplers.

\section{CONCLUSION}

This paper proposed the architecture of a directional coupler by inserting the grounding area as a capacitor to offset the reactance caused by the discontinuity in the bend connected to $50 \Omega$ transmission lines, and shaping the segments of TLs in the center of the coupler. The comparisons in Table II shows; the directivity of the proposed architecture is superior to that of the conventional one. This proves the directional coupler with a relatively high directivity of more than $-25 \mathrm{~dB}$, compared with $-20 \mathrm{~dB}$ of conventional directional couplers, can be obtained by the proposed grounding composed technic design method. Therefore, the proposed directional coupler with the directivity between 25 to $24 \mathrm{~dB}$ inside the working bandwidth is substantially higher and better performance than $20 \mathrm{~dB}$, as the practical index can be sufficiently suitable for high power very high frequency (VHF) Radar system. More studies are required to additionally improve the directivity of the proposed grounding composed architecture.

\section{REFERENCES}

[1] Pozar, David M, Microwave Engineering, 4th Edition, John Wiley \& Sons, Inc., United States of America, 2012.

[2] Inder J. Bahl, Fundamentals of RF and Microwave Transistor Amplifiers, John Wiley \& Sons, Inc., Hoboken, New Jersey, 2009.

[3] G.C.Matthaei and Leo Young, Microwave Filters, Impedance Matching Network, and Coupling Structure, Artech House, 1980.

[4] K.C. Gupta, R. Garg, and I.J. Bahl, Microstrip Lines and Slotlines, Artech House, Dedham Mass, 1979.

[5] AbdullaEroglu, Introduction to RF Power Amplifier Design and Simulation, Taylor\& Francis Group, LLC, Boca Raton, 2016.

[6] Zaiki Awang, Microwave Systems Design, Springer, New York, 2014.

[7] W. Alan Davis, Radio Frequency Circuit Design, 2th Edition, John Wiley \& Sons, Inc., United States of America,2011. 